\title{
Defining interactions between DNA-PK and ligase IV/XRCC4
}

\author{
Hsin-Ling Hsu, Steven M. Yannone, and David J. Chen* \\ Department of Cell and Molecular Biology \\ Life Sciences Division \\ Lawrence Berkeley National Laboratory \\ Berkeley, CA 94720
}

Keywords: DNA double-strand breaks; Non-homologous end joining; DNA-PK; Ku;

ligase IV/XRCC4

*Corresponding author. Tel.: 510-495-286; fax: 510-486-6816

E-mail address: djchen@lbl.gov 


\section{Abstract}

Non-homologous end joining (NHEJ) is a major pathway for the repair of DNA double-strand breaks in mammalian cells. DNA-dependent protein kinase (DNA-PK), ligase IV, and XRCC4 are all critical components of the NHEJ repair pathway. DNA-PK is composed of a heterodimeric DNA-binding component, $\mathrm{Ku}$, and a large catalytic subunit, DNA-PKcs. Ligase IV and XRCC4 associate to form a multimeric complex that is also essential for NHEJ. DNA-PK and ligase IV/XRCC4 interact at DNA termini which results in stimulated ligase activity. Here we define interactions between the components of these two essential complexes, DNA-PK and ligase IV/XRCC4. We find that ligase IV/XRCC4 associates with DNA-PK in a DNA-independent manner. The specific protein-protein interactions that mediate the interaction between these two complexes are further identified. Direct physical interactions between ligase IV and $\mathrm{Ku}$ as well as between XRCC4 and DNA-PKcs are shown. No direct interactions are observed between ligase IV and DNA-PKcs or between XRCC4 and Ku. Our data defines the specific protein pairs involved in the association of DNA-PK and ligase IV/XRCC4, and suggests a molecular mechanism for coordinating the assembly of the DNA repair complex at DNA breaks. 


\section{Introduction}

DNA damage occurs in all living cells and presents a major threat to the integrity of the genome. DNA double-strand breaks (DSBs) are particularly hazardous, as a single un-repaired break can be lethal to a cell, and incorrect repair can cause gross genetic rearrangements. Eukaryotic cells have evolved two major pathways for the repair of DNA double-strand breaks: nonhomologous end joining (NHEJ) and homologous recombination [1]. Both repair pathways require recognition of DSBs, localization of repair enzymes to the site of damage, DNA processing to yield ligatable termini, and finally ligation to reseal the break. The NHEJ pathway is strictly dependent upon functional DNA-PK, ligase IV, and XRCC4 [1-5]. The number of proteins and processes required to repair DSBs in mammalian cells suggests the existence of a mechanism for the temporal and spatial coordination of these proteins and processes.

The DNA-dependent protein kinase (DNA-PK) is a nuclear serine/threonine protein kinase and a key component of the mammalian NHEJ pathway [6,7]. DNA-PK is a member of the phosphatidyl-inositol 3 kinase (PI 3-kinase) super-family of kinases and consists of a 460-kDa catalytic subunit (DNA-PKcs) and a DNA binding component (Ku) [8,9]. $\mathrm{Ku}$ is a heterodimer comprised of $70-\mathrm{kDa}(\mathrm{Ku} 70)$ and $80-\mathrm{kDa}(\mathrm{Ku} 80)$ subunits and binds tightly to DNA termini in a sequence-independent manner. The high affinity of $\mathrm{Ku}$ 
for DNA termini and its abundance in the nuclei suggest that Ku may be involved in early recognition of DSBs $[10,11]$. The kinase activity of DNA-PK is dependent upon the assembly of DNA-PKcs with DNA-bound Ku, resulting in an active holo-enzyme/DNA complex [9]. Disruption of genes encoding DNA-PKcs, Ku70, or Ku80 results in severe defects in NHEJ and V(D)J recombination $[3,5,12,13]$. Furthermore, catalytically inactive mutants of DNA-PKcs fail to complement deficiencies in DSB repair or V(DJ) recombination in DNA-PKcs deficient cells, indicating that the kinase activity of DNAPK is required for mammalian NHEJ [13]. Although the requirement for DNA-PK and its kinase activity in NHEJ has been clearly established, the specific function(s) of DNAPK in NHEJ remain unclear. DNA-PK may function as a DNA-damage sensor, its DNAbreak dependent activation triggering an intracellular signaling pathway through phosphorylation of specific target proteins. Alternatively, DNA-PK may act directly in NHEJ by functioning as a scaffold for assembly and coordination of the repair enzymes at DNA breaks.

Ligase IV and XRCC4 form hetero-multimers and ligase IV activity is stimulated by its association with XRCC4 [14-16]. Cells deficient in XRCC4 have significantly reduced levels of ligase IV, suggesting that ligase IV may require XRCC4 for its stability [17]. A functional ligase IV/XRCC4 complex is also required for the repair of DSBs via the NHEJ pathway $[18,19]$. Several lines of evidence indicate that DNA-PK and ligase 
IV/XRCC4 functionally interact during NHEJ. Cells lacking DNA-PKcs, Ku, ligase IV, or XRCC4 are all impaired in $\mathrm{V}(\mathrm{D}) \mathrm{J}$ recombination and are hypersensitive to irradiation [5,20]. Direct interactions between ligase IV/XRCC4 and DNA-PK on DNA termini have been observed [21]. Additionally, Ku has been shown to stimulate ligase IV activity in vitro [22], possibly due to its ability to tether two DNA termini. Furthermore, Ku has been reported to recruit ligase IV/XRCC4 to DNA ends via protein-protein interactions [23], and XRCC4 can stimulate the assembly of the DNA-PK/DNA complex [24].

Here we report that the ligase activity associated with DNA-PK in cellular extracts is primarily ligase IV, and not ligase I or ligase III. We further show that ligase IV interacts directly with $\mathrm{Ku}$, and DNA-PK binds to XRCC4. These data suggest a possible mechanism for the coordination of repair enzyme assembly during NHEJ. 


\section{Materials and methods}

\subsection{Abbreviations}

Non-homologous end joining (NHEJ), DNA double-strand breaks (DSBs), DNAdependent protein kinase (DNA-PK), phosphatidyl-inositol 3 kinase (PI 3-kinase), adenylated-ligase intermediate (ligase-AMP), ethidium bromide (EtBr).

\subsection{Antibodies}

Ku80 hybridoma was a gift from Dr. N.-H. Yeh (Yang-Ming University, Taiwan). DNA-PKcs (25-4) monoclonal antibody was purchased from NeoMarkers (Fremont, CA). Rabbit $\alpha$-XRCC4 antibodies were provided by S.P. Jackson (Cambridge University, UK) or purchased (SeroTec, UK). Non-immunized mouse or rabbit IgG was purchased from Santa Cruz Biotechnology (Santa Cruz, CA). Rabbit $\alpha$-ligase IV antibody was raised against a recombinant fragment of ligase IV that was purified from E. coli. 
Human cervical carcinoma HeLa and malignant glioma M059J and M059K cells were propagated in RPMI 1640 medium (Gibco-BRL) supplemented with $10 \%$ fetal bovine serum (HyClone).

\subsection{Protein purification}

XRCC4 and ligase IV were co-expressed and purified from insect cells infected with recombinant baculovirus as described [14]. The baculoviral co-expressed $\mathrm{Ku} 70 / \mathrm{Ku} 80$ was purified as described [25]. Ku and DNA-PKcs were purified from human placenta or HeLa cells as described $[25,26]$.

\subsection{In vitro transcription/translation}

Ligase IV full-length cDNA was cloned into pET28a(+) (Novagen). Purified plasmid DNA was used for in vitro transcription/translation reactions with the TNT Coupled Reticulocyte Lysate System (Promega). Uncoupled radioactive materials were removed by passing through Nu-Clean D25 column (Shelton Scientific). 


\subsection{Cell extracts and immunoprecipitation}

Exponentially growing cells were trypsinized, washed twice with PBS, and lysed in buffer A (1\% Triton X-100, 150 mM NaCl, 1 mM EDTA, 1 mM EGTA, 50 mM Tris$\mathrm{HCl} \mathrm{pH} 7.4,1 \mathrm{mM}$ DTT, $10 \%$ glycerol, $1 \mathrm{mM}$ phenylmethylsulfonyl fluoride) $\left(3.5 \times 10^{7}\right.$ cells $/ \mathrm{ml})$. After incubation for $30 \mathrm{~min}$ at $4^{\circ} \mathrm{C}$, cell lysates were clarified by centrifugation at $10,000 \mathrm{~g}$ for $10 \mathrm{~min}$. As indicated, cell extracts were pretreated with ethidium bromide (EtBr) for $10 \mathrm{~min}$ at $4^{\circ} \mathrm{C}$ followed by centrifugation. Antibodies were added to lysates for $4 \mathrm{hr}$ at $4^{\circ} \mathrm{C}$ with gentle agitation. Thirty microliters of UltraLink ${ }^{\mathrm{TM}}$ Immobilized Protein A/G beads (Pierce) was added to reaction mixtures and incubated for $1 \mathrm{hr}$ at $4^{\circ} \mathrm{C}$. The immunobeads were washed 5 times with NET buffer (0.5\% NP-40, 1 mM EDTA, 1 mM EGTA, $150 \mathrm{mM} \mathrm{NaCl}, 5 \mathrm{mM} \mathrm{MgCl} 2,50 \mathrm{mM}$ Tris- $\mathrm{HCl} \mathrm{pH}$ 8.0) and rinsed once with reaction buffer.

\subsection{Adenyl-ligase assay}

Immune complexes were reacted for $30 \mathrm{~min}$ at $25^{\circ} \mathrm{C}$ with $25 \mu \mathrm{l}$ of ligation buffer containing $50 \mathrm{mM}$ Tris- $\mathrm{HCl} \mathrm{pH} \mathrm{8.0,10} \mathrm{mM} \mathrm{MgCl} 2,5 \mathrm{mM}$ dithiothreitol, $2 \mu \mathrm{g}$ of BSA, 2 $\mu \mathrm{Ci}$ of $\left[\alpha-{ }^{32} \mathrm{P}\right] \mathrm{ATP}(3000 \mathrm{Ci} / \mathrm{mmol})$, and $0.25 \mathrm{mM}$ ATP. Reactions were terminated by 
adding $25 \mu \mathrm{l}$ of $2 \mathrm{x}$ SDS sample buffer and heating at $95^{\circ} \mathrm{C}$ for $5 \mathrm{~min}$. Adenylated proteins were resolved by $7.5 \%$ SDS-PAGE and detected by autoradiography.

\subsection{Preparation of oligo/polynucleotide substrates}

Oligonucleotides $(r A)_{18}$ were synthesized. Oligonucleotides $(d T)_{16}$, polynucleotides (rA), and polynucleotides (dT) were purchased (Amersham Pharmacia Biotech). Oligonucleotide $(\mathrm{rA})_{18}$ was mixed with an equimolar amount of polynucleotide $(\mathrm{dT})$. Oligonucleotide $(\mathrm{dT})_{16}$ was mixed with an equimolar amount of polynucleotide (rA). The oligo/polynucleotide mixtures were incubated at $95^{\circ} \mathrm{C}$ for $10 \mathrm{~min}$ and cooled slowly to room temperature to facilitate annealing.

\subsection{De-adenylation of adenyl-ligase intermediates}

The adenylation reactions were performed as described above, and then incubated with $1 \mu \mathrm{g}$ of oligo(dT)-poly(rA) or oligo(rA)-poly(dT) substrates for $1 \mathrm{hr}$ at $25^{\circ} \mathrm{C}$. The de-adenylation reactions were resolved by SDS-PAGE and detected by autoradiography. 2.10 In vitro phosphorylation of $\mathrm{Ku}, \mathrm{XRCC4}$, XRCC4/ligase IV 
For preparation of Far-Western probes, $25 \mu \mathrm{l}$ of kinase reactions containing $1 \mu \mathrm{g}$ of purified baculoviral-expressed Ku or XRCC4, $10 \mu \mathrm{Ci}$ of $\left[\gamma_{-}{ }^{32} \mathrm{P}\right] \mathrm{ATP}(3000 \mathrm{Ci} / \mathrm{mmol}), 50$ $\mathrm{mM}$ Tris- $\mathrm{HCl} \mathrm{pH} 7.4,10 \mathrm{mM} \mathrm{MgCl}, 10 \mathrm{mM}$ dithiothreitol, and DNA-PKcs immunoprecipitated from HeLa cell lysates, were incubated for $1 \mathrm{hr}$ at $37^{\circ} \mathrm{C}$. The DNAPKcs immune beads were separated by centrifugation. Reaction mixtures were passed through a column to remove uncoupled radioactive materials.

To prepare phospho-labeled proteins for in vitro co-immunoprecipitation assays, $200 \mathrm{ng}$ of purified human placental Ku and DNA-PKcs were incubated with $50 \mu \mathrm{l}$ of dsDNA cellulose (Amersham Parmacia Biotech) in kinase buffer for $1 \mathrm{hr}$ at $4^{\circ} \mathrm{C}$. The DNA beads were then washed 4 times with kinase buffer. Two micrograms of purified recombinant XRCC4 or XRCC4/ligase IV were phosphorylated in vitro by DNA-PK assembled on DNA beads. DNA-PK/DNA beads were separated by centrifugation and the free isotope was removed from the probes as described above.

\subsection{In vitro co-immunoprecipitation assay}

${ }^{32} \mathrm{P}$-XRCC4, ${ }^{32} \mathrm{P}$-XRCC4/ligase IV, ${ }^{35} \mathrm{~S}$-ligase IV, or in vitro reconstituted ${ }^{35} \mathrm{~S}-$ ligase IV/XRCC4 were incubated with $1 \mu \mathrm{g}$ of BSA, DNA-PKcs, Ku, or Ku/DNAPKcs/DNA for $4 \mathrm{hr}$ at $4^{\circ} \mathrm{C} . \alpha-\mathrm{P} 21, \alpha$-DNA-PKcs and $\alpha$-Ku antibodies were added to 
reactions and incubated for $4 \mathrm{hr}$ at $4^{\circ} \mathrm{C}$. Protein $\mathrm{A} / \mathrm{G}$ beads were added to the reaction mix and incubated for $1 \mathrm{hr}$ at $4^{\circ} \mathrm{C}$. Immunoprecipitates were washed, eluted, and resolved by SDS-PAGE, and radiolabeled proteins were detected by phosphor imaging and analyzed by ImageQuant software (Molecular Dynamics).

\subsection{Far-Western analysis}

Two hundred nanograms of purified BSA, human DNA-PKcs, and baculoviralexpressed $\mathrm{Ku}, \mathrm{XRCC} 4$, and XRCC4/Ligase IV were spotted onto nitrocellulose. Filters were blocked in buffer containing $50 \mathrm{mM}$ Tris- $\mathrm{HCl} \mathrm{pH}$ 7.4, $150 \mathrm{mM} \mathrm{NaCl}, 0.1 \%$ Tween20 , and $5 \%$ non-fat dry milk for $1 \mathrm{hr} .{ }^{32} \mathrm{P}-\mathrm{Ku}$ or ${ }^{32} \mathrm{P}-\mathrm{XRCC} 4$ probes were reacted with the filters in blocking buffer containing $1 \%$ non-fat milk for 2 hours at $25^{\circ} \mathrm{C}$. Blots were washed 4 times for 10 min each in blocking buffer without milk. Hybridizing signals were detected by phosphor imaging.

\subsection{Immunoblotting}

Protein samples were heated with SDS sample buffer at $95^{\circ} \mathrm{C}$ for $10 \mathrm{~min}$, separated by SDS-PAGE, and electro-transferred onto a nitrocellulose membrane. Filters 
were blocked as described above, immunoblotted with primary antibodies, and subsequently probed with horseradish peroxidase-conjugated secondary antibodies. The antibody-reactive proteins were visualized by chemiluminescence (Amersham). 


\section{Results}

\subsection{DNA ligase IV co-immunoprecipitates with DNA-PK}

To determine whether ligase activity is associated with DNA-PK, we immunoprecipitated DNA-PK from cellular extracts and assayed for the presence of ligase. ATP-dependent ligases react with ATP to form a covalent bond with AMP, resulting in an adenylated-ligase intermediate (ligase-AMP) [27]. The addition of [ $\alpha$ $\left.{ }^{32} \mathrm{P}\right]$-ATP to the immunoprecipitates allows for the formation and detection of ligaseAMP intermediates by autoradiography. HeLa cell lysates were incubated with antibodies recognizing either DNA-PKcs ( $\alpha$-DNA-PKcs), Ku80 ( $\alpha-\mathrm{Ku}), \mathrm{XRCC} 4(\alpha-$ XRCC4), or IgG from normal mouse serum (Fig. 1A). The resulting immunoprecipitates were incubated with $\left[\alpha{ }^{32} \mathrm{P}\right]-\mathrm{ATP}$, and resolved by SDS-PAGE. We observed ligaseAMP intermediates associated with XRCC4, Ku80, and DNA-PKcs having an approximate molecular mass of $110 \mathrm{kDa}$ (Fig. 1A, lanes 3-5).

Human ligase I, ligase III, and ligase IV have similar molecular masses (approximately $110 \mathrm{kDa}$ ), therefore the specific ligase associated with DNA-PK cannot be distinguished by electrophoretic mobility alone. However, these three ligases have differential substrate specificities and can be identified by their ability to join different 
oligonucleotide substrates in vitro [28]. DNA ligase I will ligate oligo(rA)-poly(dT), DNA ligase III can join both oligo(rA)-poly(dT) and oligo(dT)-poly(rA), and DNA ligase IV is able to ligate oligo(dT)-poly(rA). In the presence of a suitable substrate, the respective ligase-AMP will catalyze DNA ligation and release the AMP (de-adenylation). These oligo(rA)-poly(dT) and oligo(dT)-poly(rA) substrates were used to determine the specific ligase associated with DNA-PK. Immunoprecipitates of DNA-PKcs, Ku, and XRCC4 were first allowed to form the ligase-AMP intermediate then incubated with the ligase specific oligonucleotide substrates and the level of ligase-AMP was evaluated (Fig. 1B). These results show the loss of ligase-AMP in the presence of oligo(dT)-poly(rA) and not with oligo(rA)-poly(dT). Hence, the ligase co-precipitated with DNA-PK is predominantly ligase IV.

\subsection{DNA is not required for ligase IV association with DNA-PK}

To determine whether DNA mediates the interaction between ligase IV/XRCC4 and DNA-PK, different concentrations of ethidium bromide (EtBr) were added to cell extracts to disrupt protein-DNA interactions $[29,30]$. As shown in Fig. 2, the association of ligase IV with Ku80, DNA-PKcs and XRCC4 persists in the presence of EtBr concentrations up to and including $400 \mu \mathrm{g} / \mathrm{ml}$. In contrast, the association of $\mathrm{Ku}$ and 
DNA-PKcs is disrupted by the addition of EtBr, showing that EtBr at these concentrations can disrupt the DNA-dependent assembly of DNA-PK (Fig. 2D and 2E). The interactions between ligase IV/XRCC4 and DNA-PK persist at EtBr concentrations that disrupt DNA-PK assembly, suggesting that ligase IV/XRCC4 and DNA-PK interactions are DNA-independent. Moreover, the ligase IV signal remains associated with both Ku and DNA-PKcs even at concentrations that disrupt the DNA-PK complex. Thus, the ligase IV/XRCC4 complex is associated with both Ku and DNA-PKcs independently.

\subsection{DNA-PKcs enhances ligase IV/XRCC4-Ku interactions in vivo}

We next investigated how DNA-PKcs influences Ku association with ligase IV in vivo. Using human glioma cell lines M059J, deficient for DNA-PKcs, and M059K that expresses DNA-PKcs, we found that the relative levels of DNA-PKcs, Ku80, ligase IV, and XRCC4 proteins are not grossly different between these different cell lines (Fig. 3A) [31]. As expected, ligase activity was not detected in DNA-PKcs immunoprecipitates from M059J cells (Fig. 3B, lane 5). Approximately equal amounts of ligase activity were precipitated by $\alpha-\mathrm{XRCC} 4$, indicating that the ligase IV/XRCC4 complex is intact and readily detectable in cells with or without DNA-PKcs protein (Fig. 3B, lanes 1 and 2). 
Interestingly, the ligase activity associated with $\mathrm{Ku}$ is approximately 10 -fold greater in cells expressing DNA-PKcs than in cells lacking DNA-PKcs (Fig. 3B, lanes 3 and 4). The ligase activity co-precipitated with DNA-PKcs from the M059K cells is specifically ligase IV, as evaluated by substrate specific de-adenylation (Fig. 3C). These results indicate that DNA-PKcs is either directly or indirectly influencing the ligase IV/XRCC4/Ku complex in vivo.

3.4 DNA-PKcs interacts with XRCC4, and Ku binds to ligase IV

To further define how ligase IV/XRCC4 interacts with the subunits of DNA-PK, immunoprecipitation assays were performed with purified component proteins. Recombinant XRCC4 was radiolabeled in a kinase reaction by DNA-PK immobilized on DNA-agarose beads. The ${ }^{32} \mathrm{P}-\mathrm{XRCC} 4$ was incubated with Ku, DNA-PKcs, or a mixture of Ku and DNA-PKcs, followed by immunoprecipitation with $\alpha$-Ku or $\alpha$-DNA-PKcs (Fig. 4A). The radiolabeled XRCC4 associates with DNA-PKcs independent of Ku (Fig. 4A, lane 4), while the association of XRCC4 with $\mathrm{Ku}$ is significantly greater in the presence of DNA-PKcs (Fig. 4A, lanes 3 and 5). To further investigate the interactions between DNA-PK and ligase IV/XRCC4, we phospho-radiolabeled purified ligase IV/XRCC4 complex as above. The labeled ligase IV $\beta^{32} \mathrm{P}-\mathrm{XRCC} 4$ was used in 
immunoprecipitation assays (Fig. 4B). In contrast to ${ }^{32} \mathrm{P}$-XRCC4 alone, ligase IV/ ${ }^{\beta 2} \mathrm{P}$ XRCC4 shows significant association with both Ku and DNA-PKcs (Fig. 4B, lanes 3-5). These data show that DNA-PKcs and Ku can each independently interact with ligase IV/XRCC4, while XRCC4 alone associates with DNA-PKcs but not Ku. These results are consistent with the observed association of ligase IV with DNA-PKcs under conditions that dissociate DNA-PK (Fig. 2D and 2E). Taken together, these results suggest that Ku may interact specifically with the ligase IV component of the ligase IV/XRCC4 complex while DNA-PKcs interacts directly with XRCC4.

Far-Western analysis was carried out to further define the interactions between DNA-PK and ligase IV/XRCC4. Purified recombinant Ku and XRCC4 were phosphoradiolabeled in vitro by immuno-bead-coupled DNA-PK. These radiolabeled proteins were used to probe for direct interactions with immobilized XRCC4, ligase IV/XRCC4, bovine serum albumin (BSA), Ku, and DNA-PKcs . Consistent with the co-precipitation results, ${ }^{32} \mathrm{P}$-XRCC4 interacts with immobilized XRCC4, ligase IV/XRCC4 and DNAPKcs, but not with Ku (Fig. 5A). The ${ }^{32} \mathrm{P}-\mathrm{Ku}$ interacts with DNA-PKcs and the ligase IV/XRCC4 complex but exhibits no binding to purified XRCC4 alone (Fig. 5B), suggesting that Ku interacts with ligase IV.

To directly test whether Ku and ligase IV interact, ligase IV was translated in vitro with ${ }^{35} \mathrm{~S}$-methionine in the presence or absence of purified XRCC4. These reactions 
resulted in ${ }^{35} \mathrm{~S}$-ligase IV and ${ }^{35} \mathrm{~S}$-ligase IV/XRCC4 complex. The translation products were incubated with Ku alone, DNA-PKcs alone, or a mixture of Ku, DNA-PKcs, and DNA (holo-DNA-PK). The reactions were then immunoprecipitated with antibody recognizing either Ku or DNA-PKcs (Fig. 5C). Both ${ }^{35}$ S-ligase IV and ${ }^{35}$ S-ligase IV/XRCC4 complex were associated with holo-DNA-PK (Fig. 5C, lanes 1 and 5). Ku alone also associated with ligase IV and the ligase IV/XRCC4 complex (Fig. 5C, lanes 2 and 6). In contrast, ${ }^{35} \mathrm{~S}$-ligase IV/XRCC4 co-precipitated with DNA-PKcs while ${ }^{35} \mathrm{~S}$ ligase IV alone failed to associate with DNA-PKcs (Fig. 5C, lanes 3 and 7), indicating that ligase IV interacts with $\mathrm{Ku}$ and not with DNA-PKcs. 


\section{Discussion}

In this report, we investigate ligase activity associated with DNA-PK in cellular extracts. Simultaneous detection of ligase I, ligase III, and ligase IV was made possible by the use of ligase-AMP intermediates for ligase detection. By discharging or deadenylating these intermediates with ligase-specific substrates, we find that the ligase activity associated with DNA-PK is almost exclusively ligase IV, and the other human ligases do not appreciably associate with DNA-PK in cellular extracts.

$\mathrm{XRCC} 4$, which forms a tight complex with ligase IV, is reported to have weak DNA binding activity, and the XRCC4 structure is consistent with it having DNA binding capabilities $[32,33]$. We find that addition of $\mathrm{EtBr}$, at concentrations that disrupt DNA-PK, does not significantly disrupt the interactions between ligase IV and either DNA-PKcs, Ku, or XRCC4 (Fig. 2), suggesting the interactions between ligase IV and DNA-PKcs, Ku, and XRCC4 are direct and not mediated by DNA. The DNA binding activity of XRCC4 may contribute to the overall stability of the ligase IV/XRCC4-DNAPK complex on DNA but it is not required for the individual protein interactions. Further, the dissociation of DNA-PKcs from Ku does not separate the ligase activity from either Ku or DNA-PKcs, indicating that ligase IV/XRCC4 can associate with Ku and DNA-PKcs independently. This is further supported by the persistent association of ligase IV with $\mathrm{Ku}$ in extracts from cells deficient for DNA-PKcs (M059J), although the 
levels of ligase are significantly reduced in the absence of DNA-PKcs (Fig. 3B, lane 3). This reduction may be due to the failure of the holo-complex to form without DNAPKcs, or to a reduced stability of the Ku/ligase IV/XRCC4 complex relative to the holocomplex. Thus, in cell extracts and in vitro, DNA-PKcs greatly enhances the association of ligase IV/XRCC4 with Ku, suggesting that DNA-PKcs either stabilizes the DNA-PK ligase IV/XRCC4 complex or is required for its assembly.

The NHEJ pathway has been reported to involve exonuclease, helicase, and polymerase activities [1]. Some of the proteins implicated in providing these functions include FEN-1 [34], MRE11 [35], Werner Syndrome protein (WRN) [36,37], and DNA polymerase alpha [38]. Although, the requirement for DNA-PK and ligase IV/XRCC4 in NHEJ has been clearly characterized by many genetic and biochemical studies [1-5], the exact function(s) of DNA-PK in NHEJ remains unclear. It has been suggested that DNAPK facilitates NHEJ by tethering DNA termini in close proximity [25], or that DNA-PK may function as a scaffold to recruit and assemble NHEJ enzymes to promote DNA repair [36,37]. It is clear that NHEJ repair requires many proteins and enzymatic events to function properly. The coordination of these repair enzymes, and the sequence of repair events, must be pivotal for efficient repair of DSBs. The spatial and temporal coordination of repair events is almost certainly mediated by protein-protein interactions at the site of the DSB. We show here that the ligase IV/XRCC4 complex can interact 
independently with both DNA-PKcs and Ku. We also show that there is specificity to the interactions between DNA-PK and ligase IV/XRCC4, in that Ku interacts predominantly with ligase IV while DNA-PKcs interacts more strongly with XRCC4. Additionally, in the absence of DNA-PKcs the association of ligase IV with Ku is significantly reduced, indicating that both the DNA-PKcs/XRCC4 and the Ku/ligase IV interactions are required for stable association of DNA-PK and ligase IV/XRCC4. These sets of interactions may ensure the correct orientation of the ligase molecule on the DNA-PK scaffold relative to the DNA. Alternatively, if the assembled DNA-PK complex is required for stable association of the ligase IV/XRCC4 complex, then the assembly of DNA-PK must precede the localization of ligase IV to the DSB. In this manner DNA-PK may temporally regulate the assembly of the NHEJ repair machinery. Identification of other NHEJ components and definition of their interactions with DNA-PK, will be central to a complete understanding of the molecular mechanism of NHEJ. 


\section{Acknowledgements}

We especially thank Drs. David Gilley and Janice Pluth, as well as Kevin Peet for critically reading this manuscript, and Dr. Doug Chan for providing some purified DNAPKcs. This work was supported by the U.S. Department of Energy under contract DEAC03-76SF00098 and by NIH grants AG917709, and CA50519. 


\section{Figure legends.}

Fig. 1. DNA ligase IV co-precipitates with DNA-PKcs and Ku. (A) HeLa cell extracts were immunoprecipitated with $\alpha$-DNA-PKcs, $\alpha$-Ku80, $\alpha$-XRCC4 antibodies or normal mouse IgG. Ligase activity was detected in immunoprecipitates by reaction with $[\alpha-$ $\left.{ }^{32} \mathrm{P}\right] \mathrm{ATP}$ to form an adenylated-ligase intermediate (ligase-AMP*). T4 DNA ligase is reacted as a positive control (T4 ligase-AMP*). The adenylated-ligase intermediates were separated by SDS-PAGE and visualized by autoradiography. (B) Immunoprecipitates from $\alpha$-DNA-PKcs, $\alpha$-Ku80, and $\alpha$-XRCC4 were first reacted with $\left[\alpha-{ }^{32} \mathrm{P}\right] \mathrm{ATP}$ to form the adenylated-ligase intermediates. Aliquots of these reactions were incubated with either oligo(dT)-poly(rA) or oligo(rA)-poly(dT) substrates and deadenylation was detected by autoradiography.

Fig. 2. DNA does not mediate the association of ligase IV with DNA-PKcs and Ku. (AC) HeLa cell extracts were pre-treated with increasing concentrations of EtBr before immunoprecipitation by $\alpha-\mathrm{Ku} 80, \alpha$-DNA-PKcs, $\alpha$-XRCC4, pre-immune mouse serum (NMS), or pre-immune rabbit serum (NRS). Adenyl-ligase assays were performed after immunoprecipitation and analyzed by autoradiography. (D-E) DNA-PKcs and Ku 
association was monitored by immunoblotting of immunoprecipitates with increasing EtBr concentrations.

Fig. 3. DNA-PKcs is required for stable association between Ku and ligase IV. (A) The expression levels of DNA-PKcs, Ku, ligase IV, and XRCC4 in HeLa, M059J, and M059K cells were analyzed by immunoblots. (B) M059J and M059K cell lysates were immunoprecipitated by $\alpha$-DNA-PKcs, $\alpha$-Ku80, or $\alpha$-XRCC4. Immunoprecipitates were subsequently assayed for ligase activity as described in MATERIALS AND METHODS. (C) Immunoprecipitates of DNA-PKcs from M059K were adenylated prior to incubation with oligo(rA)-poly(dT) and oligo(dT)-poly(rA). Adenylated or de-adenylated products were separated by SDS-PAGE and visualized by autoradiography.

Fig. 4. Ligase IV/XRCC4 is co-immunoprecipitated with DNA-PK in vitro. (A) XRCC4 was phosphorylated in vitro by DNA-PK immobilized on dsDNA sepharose. ${ }^{32} \mathrm{P}-\mathrm{XRCC} 4$ was incubated with $\mathrm{Ku}$ (lane 3), DNA-PKcs (lane 4), or a mixture of Ku and DNA-PKcs (lane 5). The reactions were immunoprecipitated by either $\alpha$-Ku (lanes 1, 3, and 5) or $\alpha$ DNA-PKcs (lanes 2 and 4). (B) Ligase IV $/^{32} \mathrm{P}-\mathrm{XRCC} 4$ was used to perform immunoprecipitation experiments as described in (A). The resulting immunoprecipitates 
were resolved by SDS-PAGE and visualized by phosphor imaging. Two forms of XRCC4 are noted, phosphorylated (p-XRCC4) and hyperphosphorylated (pp-XRCC4).

Fig. 5. XRCC4 binds DNA-PKcs, and Ku interacts with ligase IV in vitro. XRCC4 and Ku were phosphorylated in vitro by immuno-bead-immobilized DNA-PK. Native XRCC4, ligase IV/XRCC4, BSA, Ku, and DNA-PKcs were spotted onto nitrocellulose filters. (A) The filter was hybridized with ${ }^{32} \mathrm{P}-\mathrm{XRCC} 4$. (B) The filter was probed with ${ }^{32} \mathrm{P}-\mathrm{Ku}$. The ${ }^{32} \mathrm{P}-\mathrm{XRCC} 4$ and ${ }^{32} \mathrm{P}-\mathrm{Ku}$ interacting proteins were analyzed by phosphor imaging and ImageQuant software. (C) In vitro translated ligase IV $\left({ }^{35} \mathrm{~S}\right.$-ligase IV) or in vitro translated ligase IV with purified XRCC4 $\left({ }^{35} \mathrm{~S}\right.$-ligase IV/XRCC4) were incubated with BSA, DNA-PKcs, Ku, or a mixture of Ku, DNA-PKcs, and DNA. Reaction mixtures were immunoprecipitated by $\alpha-K u 80, \alpha$-DNA-PKcs, or $\alpha-p 21$. The immune complexes were resolved by SDS-PAGE and visualized by phosphor imaging. (D) The coomassie blue stained gels show the purity of Ku70/Ku80, DNA-PKcs, XRCC4, and ligase IV/XRCC4 proteins. 


\section{References}

[1] S.E. Critchlow, S.P. Jackson, DNA end-joining: from yeast to man, Trends Biochem Sci. 23 (1998) 394-398.

[2] R. Kanaar, J.H. Hoeijmakers, D.C. van Gent, Molecular mechanisms of DNA double strand break repair, Trends Cell Biol. 8 (1998) 483-489.

[3] P.A. Jeggo, Identification of genes involved in repair of DNA double-strand breaks in mammalian cells, Radiat Res. 150 (1998) S80-S91.

[4] Y. Tsukamoto, H. Ikeda, Double-strand break repair mediated by DNA endjoining, Genes Cells. 3 (1998) 135-144.

[5] M.R. Lieber, The biochemistry and biological significance of nonhomologous DNA end joining: an essential repair process in multicellular eukaryotes, Genes Cells. 4 (1999) 77-85.

[6] S.P. Jackson, P.A. Jeggo, DNA double-strand break repair and V(D)J recombination: involvement of DNA-PK, Trends Biochem Sci. 20 (1995) 412415.

[7] L.A. Hanakahi, M. Bartlet-Jones, C. Chappell, D. Pappin, S.C. West, Binding of inositol phosphate to DNA-PK and stimulation of double- strand break repair, Cell. 102 (2000) 721-729. 
[8] K.O. Hartley, et al., DNA-dependent protein kinase catalytic subunit: a relative of phosphatidylinositol 3-kinase and the ataxia telangiectasia gene product, Cell. 82 (1995) 849-856.

[9] S.P. Jackson, DNA-dependent protein kinase, Int J Biochem Cell Biol. 29 (1997) 935-938.

[10] P.R. Blier, A.J. Griffith, J. Craft, J.A. Hardin, Binding of Ku protein to DNA. Measurement of affinity for ends and demonstration of binding to nicks, J Biol Chem. 268 (1993) 7594-7601.

[11] W.S. Dynan, S. Yoo, Interaction of Ku protein and DNA-dependent protein kinase catalytic subunit with nucleic acids, Nucleic Acids Res. 26 (1998) 15511559.

[12] S.R. Peterson, M. Stackhouse, M.J. Waltman, F. Chen, K. Sato, D.J. Chen, Characterization of two DNA double-stranded break repair-deficient cell lines that express inactive DNA-dependent protein kinase catalytic subunits, J Biol Chem. 272 (1997) 10227-10231.

[13] A. Kurimasa, S. Kumano, N.V. Boubnov, M.D. Story, C.S. Tung, S.R. Peterson, D.J. Chen, Requirement for the kinase activity of human DNA-dependent protein kinase catalytic subunit in DNA strand break rejoining, Mol Cell Biol. 19 (1999) $3877-3884$. 
[14] K.J. Lee, J. Huang, Y. Takeda, W.S. Dynan, DNA ligase IV and XRCC4 form a stable mixed tetramer that functions synergistically with other repair factors in a cell-free end-joining system, J Biol Chem. 275 (2000) 34787-34796.

[15] S.E. Critchlow, R.P. Bowater, S.P. Jackson, Mammalian DNA double-strand break repair protein XRCC4 interacts with DNA ligase IV, Curr Biol. 7 (1997) 588-598.

[16] U. Grawunder, M. Wilm, X. Wu, P. Kulesza, T.E. Wilson, M. Mann, M.R. Lieber, Activity of DNA ligase IV stimulated by complex formation with XRCC4 protein in mammalian cells, Nature. 388 (1997) 492-495.

[17] M. Bryans, M.C. Valenzano, T.D. Stamato, Absence of DNA ligase IV protein in XR-1 cells: evidence for stabilization by XRCC4, Mutat Res. 433 (1999) 53-58.

[18] U. Grawunder, D. Zimmer, S. Fugmann, K. Schwarz, M.R. Lieber, DNA ligase IV is essential for V(D)J recombination and DNA double- strand break repair in human precursor lymphocytes, Mol Cell. 2 (1998) 477-484.

[19] U. Grawunder, D. Zimmer, P. Kulesza, M.R. Lieber, Requirement for an interaction of XRCC4 with DNA ligase IV for wild- type V(D)J recombination and DNA double-strand break repair in vivo, J Biol Chem. 273 (1998) 2470824714.

[20] P.A. Jeggo, DNA breakage and repair, Adv Genet. 38 (1998) 185-218. 
[21] L. Chen, K. Trujillo, P. Sung, A.E. Tomkinson, Interactions of the DNA ligase IV-XRCC4 complex with DNA ends and the DNA-dependent protein kinase, $\mathrm{J}$ Biol Chem. 275 (2000) 26196-26205.

[22] D.A. Ramsden, M. Gellert, Ku protein stimulates DNA end joining by mammalian DNA ligases: a direct role for Ku in repair of DNA double-strand breaks, Embo J. 17 (1998) 609-614.

[23] S.A. Nick McElhinny, C.M. Snowden, J. McCarville, D.A. Ramsden, Ku recruits the XRCC4-ligase IV complex to DNA ends, Mol Cell Biol. 20 (2000) 29963003.

[24] R. Leber, T.W. Wise, R. Mizuta, K. Meek, The XRCC4 gene product is a target for and interacts with the DNA- dependent protein kinase, J Biol Chem. 273 (1998) 1794-1801.

[25] R.B. Cary, S.R. Peterson, J. Wang, D.G. Bear, E.M. Bradbury, D.J. Chen, DNA looping by Ku and the DNA-dependent protein kinase, Proc Natl Acad Sci U S A. 94 (1997) 4267-4272.

[26] D.W. Chan, R. Ye, C.J. Veillette, S.P. Lees-Miller, DNA-dependent protein kinase phosphorylation sites in Ku 70/80 heterodimer, Biochemistry. 38 (1999) 1819-1828. 
[27] A.J. Doherty, S.W. Suh, Structural and mechanistic conservation in DNA ligases, Nucleic Acids Res. 28 (2000) 4051-4058.

[28] P. Robins, T. Lindahl, DNA ligase IV from HeLa cell nuclei, J Biol Chem. 271 (1996) 24257-24261.

[29] J.S. Lai, W. Herr, Ethidium bromide provides a simple tool for identifying genuine DNA- independent protein associations, Proc Natl Acad Sci U S A. 89 (1992) 6958-6962.

[30] A. Suwa, M. Hirakata, Y. Takeda, S.A. Jesch, T. Mimori, J.A. Hardin, DNAdependent protein kinase (Ku protein-p350 complex) assembles on doublestranded DNA, Proc Natl Acad Sci U S A. 91 (1994) 6904-8.

[31] S.P. Lees-Miller, R. Godbout, D.W. Chan, M. Weinfeld, R.S. Day, G.M. Barron, J. Allalunis-Turner, Absence of p350 subunit of DNA-activated protein kinase from a radiosensitive human cell line, Science. 267 (1995) 1183-1185.

[32] M. Modesti, J.E. Hesse, M. Gellert, DNA binding of Xrcc4 protein is associated with V(D)J recombination but not with stimulation of DNA ligase IV activity, Embo J. 18 (1999) 2008-2018.

[33] M.S. Junop, M. Modesti, A. Guarne, R. Ghirlando, M. Gellert, W. Yang, Crystal structure of the Xrcc4 DNA repair protein and implications for end joining, Embo J. 19 (2000) 5962-70. 
[34] M.R. Lieber, The FEN-1 family of structure-specific nucleases in eukaryotic DNA replication, recombination and repair, Bioessays. 19 (1997) 233-40.

[35] T.T. Paull, M. Gellert, The 3' to 5' exonuclease activity of Mre 11 facilitates repair of DNA double-strand breaks, Mol Cell. 1 (1998) 969-79.

[36] M.P. Cooper, A. Machwe, D.K. Orren, R.M. Brosh, D. Ramsden, V.A. Bohr, Ku complex interacts with and stimulates the Werner protein, Genes and Development. 14 (2000) 907-12.

[37] B. Li, L. Comai, Functional interaction between $\mathrm{Ku}$ and the werner syndrome protein in DNA end processing, J Biol Chem. 275 (2000) 28349-28352.

[38] L. Islas, C.F. Fairley, W.F. Morgan, DNA synthesis on discontinuous templates by human DNA polymerases: implications for non-homologous DNA recombination, Nucleic Acids Res. 26 (1998) 3729-38. 